\title{
Multi-Harmonic Optical Comb Generation
}

\author{
S. J. Fabbri, S. Sygletos, A. D. Ellis \\ Photonic Systems Group, Tyndall National Institute and Physics Department of University College \\ Cork, Lee Maltings, Cork, Ireland \\ simon.fabbri@tyndall.ie
}

\begin{abstract}
We present a novel optical comb generation technique based on the use of a multi-harmonic electrical signal for driving the MZM. The proposed scheme is highly power efficient and gives rise to square shaped combs of advanced flatness and side mode suppression ratio while maintaining a stable performance over a long time period
\end{abstract}

\section{Introduction}

Optical comb generation is a topic of intense interest due to its wide application range in different areas of photonic technology. Of particular importance is its potential role in the development transmitter units for advanced multi-carrier signal formats, i.e. orthogonal frequency division multiplexed signals (OFDM) in next generation optical networks [1]. Apart from increasing the attainable spectral density, it may also bring significant cost and power consumption benefits as well as simpler system designs by reducing the number of temperature controlled laser sources and wavelength lockers required. For this application field any potential comb generation technique should concentrate a number of key merits. It should produce high coherent and stable optical combs of square shape and in a power efficient way, with uniform amplitudes for the wanted comb lines, and excellent intrinsic suppression of the unwanted comb lines. The corresponding configuration should be also simple, cost effective and compact, enabling subsequent integration.

Not many of the techniques that have been proposed in literature meet the aforementioned requirements. For example, mode-locking, of semiconductor [2] or Er:Yb: glass lasers [3] can provide a good optical signal-to-noise ratio (OSNR), however, they require equalizing filters to enable reasonable flatness, and the line spacing of the produced comb is fixed by the cavity length. Techniques based on highly nonlinear fibers by using four-wave mixing effects [4] may enable combs of wide optical bandwidth, but they are bulky and require optical power pumps. Alternatively, the use of external Mach-Zehnder modulators (MZM) has recently demonstrated very attractive features for the development of future transmitter units [5-8]. Techniques of this family, apart from their advanced flatness, low jitter and stable performance, offer also flexibility in the control of the line spacing, independence in the choice of the central wavelength, as well as the potential for integration. The frequency response and the tolerance in the RF driving power of the modulator limit the number of lines that can be generated from a single stage. However, making use of cascaded MZM configurations [7] optical combs of larger bandwidth and number of lines can be produced.

Up to date, to achieve advanced flatness performance over an increased line number required strong RF powers levels driving the $M Z M$. This brings a significant efficiency issue as a lot of power goes to a large number of unwanted sideband optical frequencies giving a "bell" shape to the generated comb. The result of this effect is also the need of an advanced filter structure, i.e. a wavelength selective switch (WSS), to square the shape of the comb which will elevate the cost and the complexity of a future transmitter unit.

In this paper we introduce a new concept, up to our knowledge, to drive the MZM by using a multi-harmonic electrical signal to generate an optical comb of high quality in terms of flatness

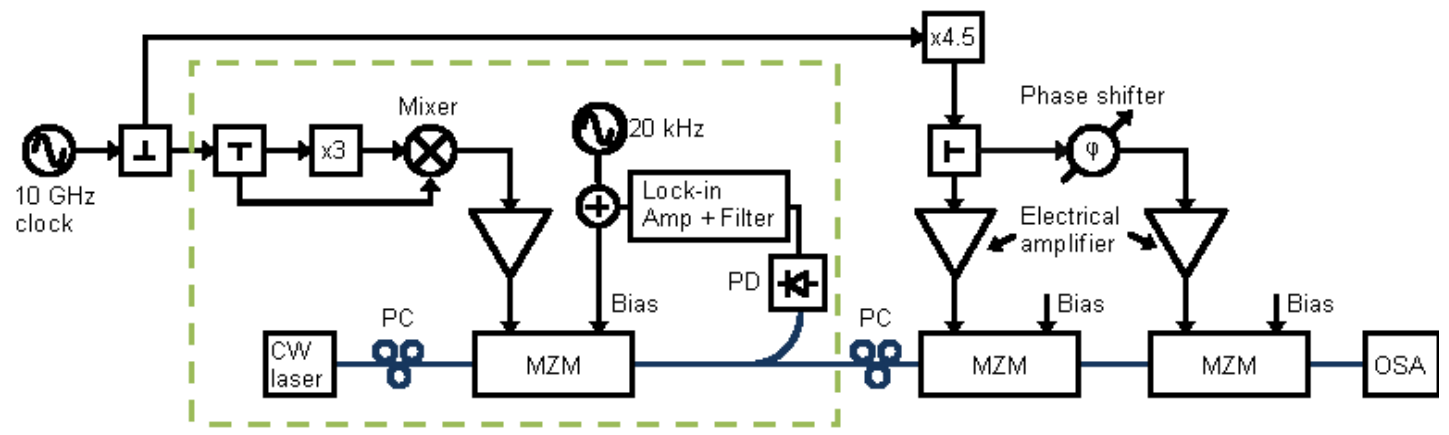

Fig. 1: Experimental setup of the optical comb generator 


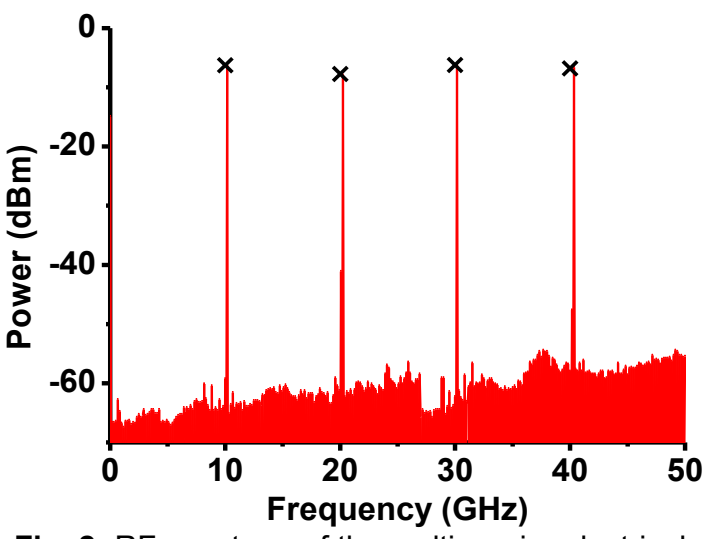

Fig. 2: RF spectrum of the multi-carrier electrical signal applied to the $1^{\text {st }} \mathrm{MZM}$ of the setup

and spectral shape from a single stage. When combined with cascaded MZM modulators, it was possible to extend the comb bandwidth, whilst maintaining the same advanced optical performance characteristics. The scheme was self-stabilized to maintain a reliable performance over a long time period.

\section{Experimental Setup and Results}

The implemented frequency comb generation scheme is illustrated in Fig. 1. It consisted of three cascaded MZM modulators of which the first modulator was driven by an electrical comb of four equally spaced frequency components at $10 \mathrm{GHz}$. To create the electrical comb a single $10 \mathrm{GHz}$ oscillator was used. Part of its emitted RF signal was multiplied by a factor of 3 to obtain the $30 \mathrm{GHz}$ component, which was subsequently mixed with the other $10 \mathrm{GHz}$ part to create RF signals at $20 \mathrm{GHz}$ and $40 \mathrm{GHz}$. The multi-harmonic signal was then amplified and applied to the optical modulator. Fig. 2 depicts the corresponding RF spectrum. Its four frequency components at $10,20,30$ and $40 \mathrm{GHz}$ had power levels of $-6.28,-7.73,-6.23$ and -6.81 $\mathrm{dBm}$, respectively. Its flatness was controlled by the power level of the $10 \mathrm{GHz}$ source to counterbalance the roll-off in the frequency response of the data modulator used $\left(\Delta f_{3 \mathrm{~dB}}\right.$ $\sim 25 \mathrm{GHz}$ ). The $\mathrm{V}_{\pi}$ of the modulator was 3.3Volts.

The proposed concept to drive the MZM with a multi-carrier electrical signal for creating the optical comb is significantly more power efficient than using a single frequency carrier. Indeed, for the latter case $\sim 1 \mathrm{~W}$ RF driving power levels have been reported [9], whilst for our case the total electrical RF driving power of the MZM did not exceed $-0.7 \mathrm{dBm}$. This was because each harmonic of the electrical comb needed to generate just a single pair of sideband frequencies in the optical domain, and therefore they could be of considerably low power. Consequently, the resulting optical comb at the

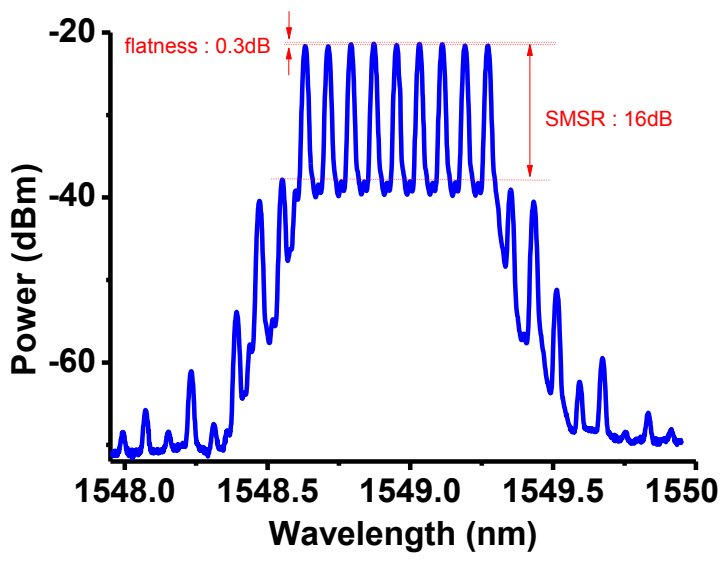

Fig. 3: Optical spectrum of the 9-line comb taken at the final output of the $1^{\text {st }}$ modulator of the setup

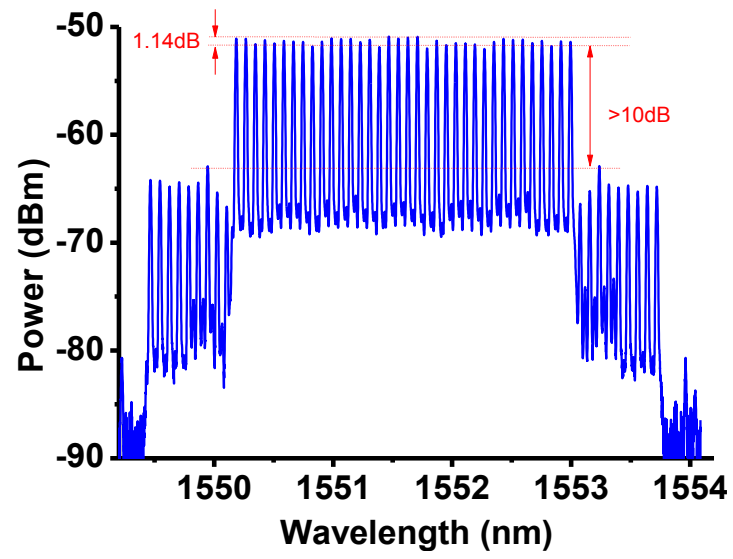

Fig. 4: Optical spectrum of the 36-line comb taken at the final output of the setup

output of the modulator consisted of 4 pairs of sideband components around the seeding laser frequency, giving 9-lines in total. The corresponding optical spectrum is depicted in Fig. 3. The generated optical comb presented a maximum power difference between the channels (flatness) of only $0.3 \mathrm{~dB}$ and a sidemode suppression ratio (SMSR) better than $16 \mathrm{~dB}$. Such advanced SMSR performance is reported for the first time in this paper and was attributed to the low power driving conditions of the MZM by the multi-harmonic electrical signal. In the proposed scheme the bandwidth of the $\mathrm{MZM}$, and not the tolerance in RF driving power, limited the number of $10 \mathrm{GHz}$ lines that could be generated from a single stage. Future transceiver units, however, will require wider comb bandwidths and larger carrier number to enable high capacity super-channel transmission. In our case, we made use of a second comb, consisting of two cascaded amplitude MZMs ( $\mathrm{V}_{\pi}$ : 3.7Volts, 3.3Volts) to replicate by multiple times the input 9-line optical signal in a wider frequency band. The two comb architectures were synchronized as the $45 \mathrm{GHz}$ 


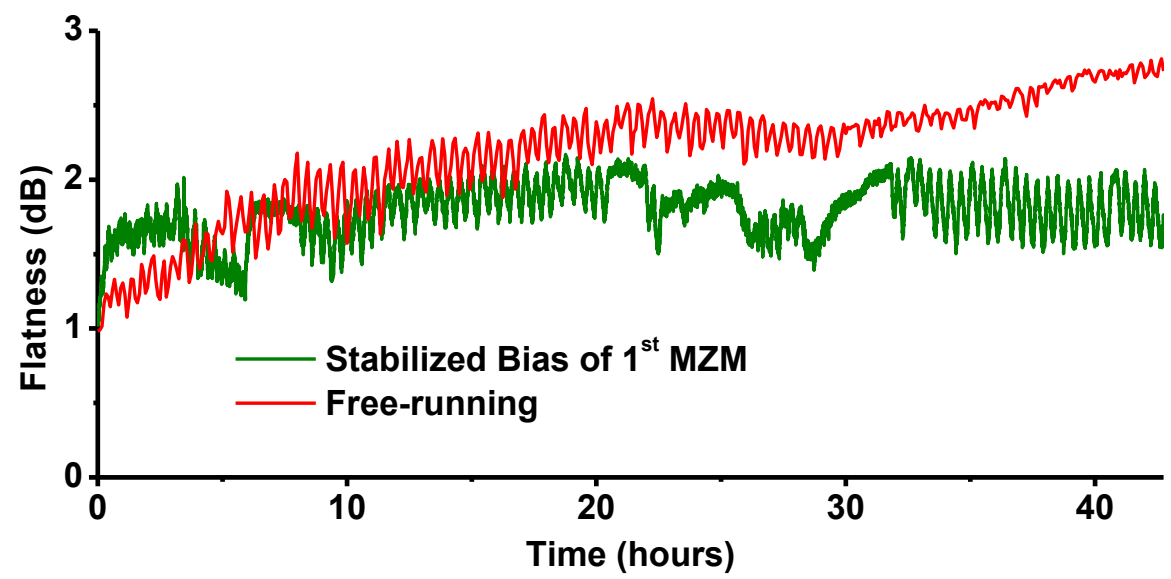

Fig. 5: Long time measurement of the flatness of the 36 line comb when the bias point of the $1^{\text {st }} \mathrm{MZM}$ is controlled by an external feedback loop (green line), and when it is free running (red line)

RF driving signal of the last two MZMs had been produced with the help of a $4.5 x$ multiplier from the common $10 \mathrm{GHz}$ clock. The combination of these two comb generator techniques delivered a 36 line optical comb with $10 \mathrm{GHz}$ interval with a flatness of $1.14 \mathrm{~dB}$ and a SMSR better than 10 $\mathrm{dB}$. The corresponding optical spectrum is depicted in Fig. 4.

Maintaining an advanced and stable flatness performance for the optical comb over a long time period is critical for the exploitation of the proposed scheme in future transmitter circuits. In our case the principal factor affecting the comb flatness was found to be the balance between the arms of the $1^{\text {st }}$ MZM, mainly acting on the central carrier in the 9-line comb. Therefore, a low speed feedback loop circuit had been implemented to control the bias point of the modulator and to mitigate any flatness fluctuations over time. To create a suitable error signal for this case, we made use of a low frequency pilot tone $(\sim 20 \mathrm{kHz})$ added on the bias control and subsequently extracted by a lock-in amplifier from the received power signal at the output of the modulator.

Fig. 5 depicts the measured flatness of the 36line comb versus time when the stabilization scheme was active as well as for the free running operation case. The total observation time was more than 40 hours and measurements have been taken periodically every 15 seconds. Under free running operation of the $1^{\text {st }}$ MZM bias point we noticed the central frequency of the 9-line comb shifting slowly out of the limits while the other lines maintained an equal power level. This also degraded the flatness of the 36-line comb which exceeded $2.8 \mathrm{~dB}$. By activating the control loop the degradation was mitigated and the overall flatness was kept bellow $2 \mathrm{~dB}$. The faster variations, with a period of 25 minutes, observed in the flatness performance are attributed to the room temperature regulation affecting the gain performance of the driving RF amplifiers. We note that the biasing as well as the RF driving conditions on the other two modulators did not affect the long term stability of the final comb.

\section{Conclusions}

In this paper we have developed a new technique for optical comb generation based on driving the MZM with a multi-harmonic electrical signal. The proposed scheme is highly power efficient as it requires RF driving power of less $0.7 \mathrm{dBm}$ to generate a 9-line comb spaced at $10 \mathrm{GHz}$ with high flatness $(0.3 \mathrm{~dB})$ and square shape (SMSR : 16dB). By combining the technique with a conventional architecture of two cascaded MZMs, 36 optical frequency carriers were generated with the same spacing and with advanced spectral characteristics of flatness, $1.14 \mathrm{~dB}$, and SMSR > 10dB. By stabilizing the scheme, we managed to maintain the high performance merits over a long time period ( $>40$ hours).

\section{Acknowledgments}

The authors are thankful to Dr. Fatima Gunning for her critical comments on the manuscript. The work has been supported by Science Foundation Ireland under the grant number $06 / 1 \mathrm{~N} / 1969$.

\section{References}

[1] F.C.G. Gunning et al, in Proc OFC 2010, OthD1

[2] S. Arahira et al, Opt. Lett. 19, 834 (1994)

[3] M. Nakazawa et al., Electron. Lett., 33, 1318 (1997)

[4] V. R. Supradeepa et al., in Proc. OFC 2011, OMQ3

[5] T. Healy et al., Optics Express 15, 2981 (2007)

[6] W. Mao et al., in Proc OFC 2004, (2004)

[7] M. Fujiwara et al., JLT 21, 2705-2714 (2003)

[8] I. Morohashi at al., in Proc ECOC 2010, P2.16

[9] R. Wu et al., Optics Letters 19, 3234, 2010 